\title{
Differences in proteoglycan deposition in the airways of moderate and severe asthmatics
}

\author{
L. Pini*, Q. Hamid*, J. Shannon*, L. Lemelin*, R. Olivenstein*, P. Ernst*, C. Lemière*, \\ J.G. Martin* and M.S. Ludwig*
}

ABSTRACT: Excess deposition of proteoglycans (PGs) has been described in the subepithelial layer of the asthmatic airway wall. However, less is known about deposition in the airway smooth muscle (ASM) layer, and whether the pattern of deposition is altered depending upon disease severity.

Endobronchial biopsies were performed in patients with severe or moderate asthma (defined using American Thoracic Society criteria) and in control subjects. Biopsies were immunostained for the PGs biglycan, lumican, versican and decorin. PG deposition was measured in the subepithelial and ASM layers, the former by calculating the area of positive staining, and the latter by determining the percentage area stained using point counting.

Immunostaining for PGs was prominent in biopsies from both moderate and severe asthmatics, compared with control subjects. While there was no difference in the amount of PG in the subepithelial layer between the two asthmatic groups, the percentage area of biglycan and lumican staining in the ASM layer was significantly greater in moderate versus severe asthmatics.

Differences in the deposition of proteoglycans within the airway smooth muscle layer of moderate versus severe asthmatics potentially impact on the functional behaviour of the airway smooth muscle in these two groups of patients.

KEYWORDS: Airway smooth muscle, biglycan, decorin, lumican, versican

$\mathbf{R}$ emodelling of the airway wall is well described in asthmatic patients [1]. Studies have shown thickening of the reticular basement membrane and increased matrix deposition in the subepithelial layer of the conducting airways [2-5]. Collagen and glycoproteins, such as fibronectin, account for some of these changes; proteoglycans (PGs) also contribute to the remodelling [6]. The precise link between remodelling and asthma severity has not yet been established. BENAYOUN et al. [7] noted an increase in collagen type III deposition in the bronchial submucosa of patients with severe, persistent asthma. A positive relationship has been demonstrated between airway wall thickening and asthma severity [5]. Conversely, CHU et al. [3] were unable to document differences in collagen type III immunostaining in airways from patients with asthma of varying severity. Furthermore, NiImI et al. [8] showed that airway wall thickness, as assessed by computerised tomography, and airway reactivity were inversely correlated. The possibility that other extracellular matrix (ECM) molecules, such as PGs, are important in contributing to asthma severity has not been investigated; nor has the question of whether matrix deposition is altered in the smooth muscle layer in moderate versus severe asthmatics been addressed.

PGs are macromolecules consisting of a protein core and glycosaminoglycan (GAG) side-chains. Different subclasses of PGs have been described, including hyalectins, such as versican, which are large molecules with many GAG side-chains; and small leucine-rich PGs, e.g. decorin, biglycan and lumican. PGs fulfil a number of important biological functions [9]. Versican, owing to the high ionic charge of its multiple GAG sidechains, plays a critical role in determining the water content of extracellular matrices, and influences tissue viscoelastic behaviour. Decorin and biglycan bind to collagen and affect collagen fibrillogenesis and matrix assembly. These molecules also bind different growth factors, such as transforming growth factor (TGF)- $\beta$ and fibroblast growth factor, and modulate their ability to influence cell proliferation and matrix deposition [10].
AFFILIATIONS

${ }^{*}$ Meakins-Christie Laboratories, Montreal Chest Institute, McGill University Hospital Centre, and \#Hôpital du Sacré-Coeur de Montréal, Université de Montréal, Montreal, QC, Canada.

CORRESPONDENCE

M.S. Ludwig

Meakins-Christie Laboratories

3626 St. Urbain Street

Montreal

QC

$\mathrm{H} 2 \mathrm{X} 2 \mathrm{P} 2$

Canada

Fax: 15143987483

E-mail: mara.ludwig@mcgill.ca

Received:

April 202005

Accepted after revision:

August 242006

SUPPORT STATEMENT

This study was supported by the Richard and Edith Strauss Foundation (Montreal, Canada), the Canadian Institutes of Health Research (Ottawa, Canada), the JT Costello Memorial Research Fund (Montreal, Canada) and GlaxoSmithKline (Mississauga, Canada). 
ROBERTS [11] observated that, in patients dying of asthma, the airway wall showed prominent staining for biglycan, decorin and versican. The present authors have shown, in endobronchial biopsy specimens obtained from mild asthmatics, that deposition of versican, biglycan and lumican was increased in the subepithelial layer of the airway wall, compared with biopsies obtained from normal volunteers [6]. REDINGTON et al. [12] showed co-localisation of decorin and TGF- $\beta_{1}$ in the subepithelial layer of the airway wall; however, there was no difference in the overall pattern of deposition between biopsy specimens from asthmatic and control subjects. A recent report in mild asthmatic subjects showed a decrease in decorin levels in the lamina propria compared with normal controls; conversely, biglycan levels were increased [13]. The nature of PG deposition in the asthmatic airway wall is thus far from clear.

The increased PG expression in the asthmatic airway wall could have important functional consequences. Thickening of the airway wall may lead to increased airway resistance because of decreases in luminal diameter, and to increased airway responsiveness because of the effects of a thickened airway wall on airway smooth muscle (ASM) shortening and subsequent airway narrowing [14]. In a study of mild atopic asthmatics, PG deposition in the subepithelial layer was positively correlated with airway responsiveness [6]. Theoretically, however, increased matrix deposition in the smooth muscle layer itself could result in decreased smooth muscle constriction $[15,16]$. A recent review by MCPARLAND et al. [15] promoted the hypothesis that remodelling has modulating effects on airway narrowing; stiffening of the airway wall could lead to decreased compressibility, while increased matrix within the smooth muscle layer could increase the impedance to ASM shortening. Much would depend on the precise mechanical characteristics of the deposited matrix. Hence, the site of enhanced matrix deposition and the specific mechanical properties of the matrix itself may be critical determinants of the impact of remodelling on airway physiology.

In the present study, in order to examine changes in PG distribution, and the contribution of PGs to airway wall remodelling in moderate and severe asthmatic patients, endobronchial biopsies were used to obtain specimens of the airway wall, and PG deposition in the different layers of the airway wall sampled was characterised by immunohistochemistry.

\section{METHODS}

\section{Participants}

A total of 27 asthmatic patients (age range 22-66 yrs) and six control subjects (age range 20-63 yrs) participated in the present study (table 1). Asthmatics of both sexes (14 severe and 13 moderate) were recruited from the Difficult Asthma Study, conducted at the Montreal Chest Institute, McGill University, and at the Hôpital du Sacré-Coeur, Université de Montréal (both Montreal, QC, Canada). Standard pulmonary function testing was performed on all subjects.

Asthma was diagnosed by a respirologist and was confirmed by an improvement in forced expiratory volume in one second (FEV1) of $\geqslant 12 \%$, with an absolute increase of $\geqslant 200 \mathrm{~mL}$ from baseline, after administration of a $\beta$-adrenergic agonist; or by a $\geqslant 20 \%$ improvement in FEV1 after a steroid trial.

Severe asthma was defined according to criteria adapted from an American Thoracic Society workshop on refractory asthma [17]. Severe asthmatic patients had either treatment with daily oral steroids for $>50 \%$ of the previous 12 months, or treatment with high-dose inhaled steroid $(>1,000 \mu \mathrm{g}$ fluticasone or equivalent per day) and at least one other drug (long-acting $\beta$-agonist, leukotriene receptor antagonist or theophylline) continuously over the previous 12 months. In addition, they had to meet at least two of the following criteria: daily use of short-acting $\beta$-agonist; persistent airflow obstruction as documented with pre-bronchodilator FEV1 $<70 \%$ and FEV1/forced vital capacity ratio $<80 \%$ predicted; at least one urgent care visit in the previous 12 months; three or more courses of oral steroids in the previous 12 months; prompt deterioration with $<25 \%$ dose reduction of steroids; or near-fatal asthma event within the previous 3 yrs.

Moderate asthmatics met all of the following criteria: wellcontrolled asthma with $200-1,000 \mu \mathrm{g} \cdot \mathrm{day}^{-1}$ of fluticasone or equivalent, with or without the use of a long-acting $\beta$-agonist; no more than two oral steroid courses in the previous 12 months but none in the past 3 months and with the total number of days on oral steroids not $>30$ days in the previous 12 months; FEV $1>70 \%$ pred and $>90 \%$ of personal best from the previous 2 yrs; and no more than one nonscheduled urgent care or office visit in the previous 12 months.

Individuals were excluded if they had other known pulmonary disease or major comorbid disease that might affect asthma disease activity, such as HIV, metastatic cancer or congestive heart failure. Informed consent was obtained before bronchoscopy was performed. The study was approved by the ethics committees of the McGill University Health Centre and the Research Centre of the Hôpital du Sacré-Coeur, Université de Montréal.

\section{Procedures}

Bronchoscopy was performed according to standard procedures. A total of 4-6 biopsy specimens per patient were taken at the carina of segmental bronchi using cup forceps. Biopsy specimens were placed in Tris-buffered saline solution and immediately transported to the laboratory for processing. For biglycan, lumican and decorin immunostaining, tissues were fixed in $4 \%$ paraformaldehyde and embedded in paraffin. Tissues were then sectioned (5- $\mu \mathrm{m}$ thickness) and placed on glass slides coated with poly-L-lysine $(0.1 \%)$ and baked overnight at $37^{\circ} \mathrm{C}$. For versican immunostaining, tissues were snap-frozen in isopentane, blocked in optimum-cutting-temperature embedding medium, sectioned (5- $\mu \mathrm{m}$ thickness) and then fixed in acetone/methanol. Some of the biopsy specimens thus obtained were used in another study [18]. Only asthmatic subjects had tissue stained for versican, as frozen biopsy specimens were not available in control subjects.

\section{Immunohistochemistry}

Slides were stained with primary antibodies to biglycan, (polyclonal rabbit, anti-human, 1:500), lumican (polyclonal rabbit, anti-human, 1:500; both gifts of P. Roughley, Shriners Hospital, McGill University), decorin (polyclonal rabbit, 
TABLE 1 Patient characteristics and spirometry

\begin{tabular}{|c|c|c|c|c|c|c|}
\hline & Sex & Age yrs & FEV 1 L & FEV $1 \%$ pred & FEV $_{1} /$ FVC & Smoking pack-yrs \\
\hline \multicolumn{7}{|c|}{ Severe asthma } \\
\hline 2 & M & 45 & 2 & 51 & 66 & 15 \\
\hline 3 & $\mathrm{~F}$ & 36 & 2.9 & 88 & 66 & 5 \\
\hline 4 & M & 52 & 1.4 & 41 & 40 & 0 \\
\hline 7 & $\mathrm{~F}$ & 59 & 1 & 48 & 73 & 0 \\
\hline 8 & M & 66 & 1.2 & 44 & 76 & 20 \\
\hline 9 & M & 51 & 3.6 & 92 & 67 & 0 \\
\hline 10 & M & 53 & 1.9 & 55 & 54 & 0 \\
\hline 11 & M & 55 & 1.9 & 58 & 66 & 20 \\
\hline 12 & $\mathrm{~F}$ & 40 & 1.0 & 33 & 47 & 0 \\
\hline \multicolumn{7}{|c|}{ Moderate asthma } \\
\hline 1 & $M$ & 49 & 2.5 & 67 & 70 & 5 \\
\hline 2 & $\mathrm{~F}$ & 50 & 2.2 & 82 & 72 & 3 \\
\hline 3 & $\mathrm{~F}$ & 61 & 2 & 100 & 76 & 20 \\
\hline 4 & $\mathrm{~F}$ & 55 & 2.1 & 84 & 72 & 0 \\
\hline 5 & M & 55 & 3.1 & 77 & 78 & 10 \\
\hline 6 & $\mathrm{~F}$ & 23 & 2.9 & 95 & 73 & 0 \\
\hline 7 & M & 42 & 3.1 & 78 & 62 & 12 \\
\hline 8 & M & 60 & 2.5 & 74 & 63 & 0 \\
\hline 9 & $\mathrm{~F}$ & 47 & 2.5 & 85 & 79 & 2 \\
\hline 10 & M & 49 & 4.4 & 119 & 82 & 0 \\
\hline 11 & M & 55 & 5.2 & 121 & 74 & 0 \\
\hline 4 & $\mathrm{~F}$ & 22 & 3.38 & 107 & 81 & 0 \\
\hline 5 & $\mathrm{~F}$ & 20 & 3.31 & 100 & 95 & 0 \\
\hline 6 & M & 28 & 4.7 & 121 & 88 & 0 \\
\hline Mean \pm SE & & 34 & $3.96 \pm 0.5$ & $109.8 \pm 7.1$ & $83.5 \pm 3.5$ & \\
\hline
\end{tabular}

FEV1: forced expiratory volume in one second; \% pred: \% predicted; FVC: forced vital capacity; F: female; M: male.

anti-mouse, 1:1000; Developmental Studies Hybridoma Bank, Iowa City, IA, USA) or versican (monoclonal mouse, antihuman, 1:500; Developmental Studies Hybridoma Bank). Secondary antibodies used were swine antibody to rabbit immunoglobulin (1:100; Dako, Glostrup, Denmark) or rabbit antibody to mouse immunogloblulin (1:60; Dako; versican staining). Steptavidin-alkaline phosphatase (1:200; Dako) or mouse alkaline phosphatase/anti-alkaline phosphatase (1:60; Dako; versican staining) was used for detection. All immunostaining was developed with Fast Red (Sigma-Aldrich, Toronto, ON, Canada). Slides were counterstained with Gill II haematoxylin and coverslips applied. Positive staining appeared red under bright-field illumination. For negative controls, sections were processed in the absence of primary antibody. Not all biopsy samples were stained for all PGs. The number of biopsy specimens examined for each PG ranged from four to six. Some patients had separate biopsy samples stained for more than one PG.

\section{Morphometry}

The slides were examined using an Olympus light microscope (Carson Group, Markam, ON, Canada) and images captured with commercial software (Image-Pro Plus; Media Cybernetics, Silver Spring, MD, USA). To detect positive staining in the subepithelial layer, $200 \times$ magnification was used. The subepithelial layer was defined as including the reticular basement 
membrane and extending down to the smooth muscle layer. Blood vessels and mucous glands were excluded from the analysis. All the subepithelial area under identifiable basement membrane in the biopsy specimen was included in the analysis. Positive staining was established by determining a colour threshold, and was quantified either by tracing the area using a digital pen (Wacom, Vancouver, WA, USA) or using image analysis software (control subjects). Area (in $\mu \mathrm{m}^{2}$ ) was standardised for the basement membrane length squared (basement membrane length was squared so that an area was corrected for an area). Values were calculated using SigmaScan (Jandel Scientific, Corte Madera, CA, USA). To detect positive staining in the smooth muscle layer, an eyepiece graticule was applied. At $100 \times$ magnification, the area of the graticule was $1.0 \mathrm{~mm}^{2}$. The amount of PG within or overlying the smooth muscle layer was expressed as the percentage of positive staining overlapping the cross-points of a 121-point grid. Measurements were made at a magnification of $1,000 \times$ in 10 randomly chosen fields per biopsy. Areas where ASM was clearly identified were included, and adjacent connective tissue was excluded.

Different morphometric techniques were used to measure PG in the subepithelial and smooth muscle layers for several reasons. When there is a well-defined area of positive staining, as was the case in the subepithelial layer, the area can be measured and standardised to basement membrane. When the area of positive staining is more diffuse, as was the case in the ASM layer, it is more difficult to measure total area. As the reference volume was well defined, an area ratio estimate (point counting) was used. PG results between the subepithelial and smooth muscle layers were not compared directly. Rather, the same technique was used to compare the signal between moderate and severe asthmatics.

\section{Statistical analysis}

Comparisons of PG deposition among severe and moderate patients and normal controls were performed using ANOVA and Bonferroni's multiple comparison test. Where data were not normally distributed, a Kruskal-Wallis test, followed by Dunn's multiple comparison test, was applied. A p-value $<0.05$ was considered statistically significant. Data are presented as mean \pm SE.

\section{RESULTS}

Characteristics of the 27 asthmatic patients (14 with severe asthma and 13 with moderate asthma) and the six control subjects are shown in table 1 . Mean FEV1 \% pred in the severe, moderate and control groups was $63.6 \pm 6.8,87.5 \pm 4.7$ and $109.8 \pm 7.1 \%$, respectively.

\section{Immunohistochemistry}

Positive staining for biglycan, lumican, versican and decorin was identified in all airway specimens of both severe and moderate asthmatic patients. Positive staining for biglycan, lumican and versican was present in both the subepithelial and the smooth muscle layers; however, the pattern of staining differed between the two populations (fig. 1a-c). Whereas staining for biglycan, lumican and versican was prominent in the smooth muscle layer in moderate asthmatics (fig. 1b), staining for PGs within the smooth muscle layer was relatively sparse in severe asthmatics (fig. 1a and c), although it was still
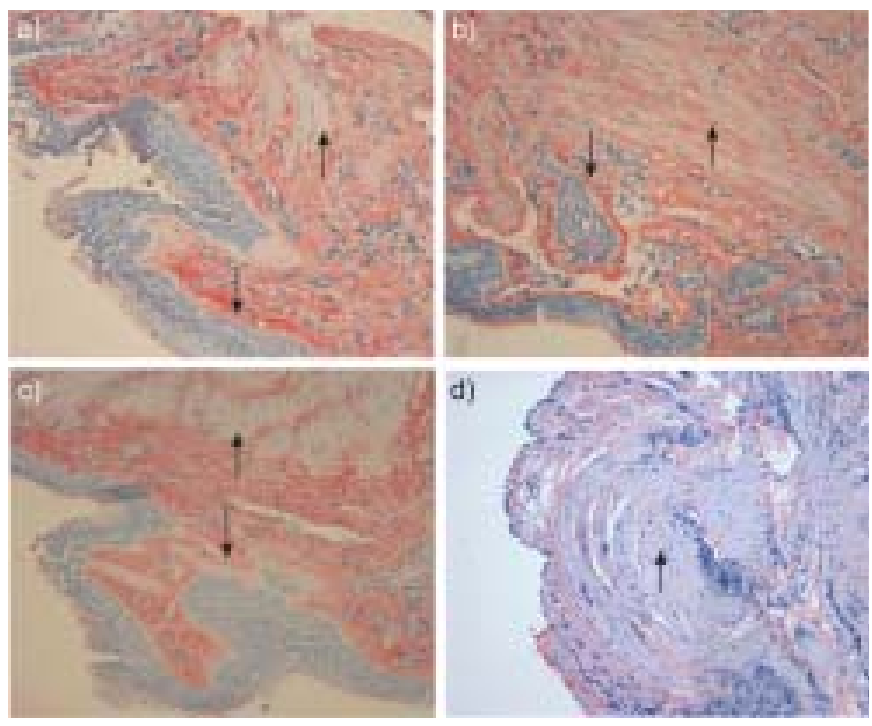

FIGURE 1. Immunohistochemical staining for biglycan ( $a, b$ and $d$ ) and lumican (c) in airway biopsies of patients with severe (a and c) and moderate (b) asthma, and a control subject (d). In the biopsy specimens from severe asthmatic patients, biglycan and lumican immunostaining was sparse within the smooth muscle layer (arrowheads) but prominent within the subepithelial layer (arrows). In the biopsy specimen stained for biglycan from the moderate asthmatic patient, biglycan deposition was evident throughout both the smooth muscle (arrowhead) and subepithelial (arrow) layers. Biglycan immunostaining was relatively absent from the smooth muscle layer in the biopsy sample from the control subject (arrowhead).

greater than that observed in control subjects (fig. 1d). Staining for these PGs within the subepithelial layer was marked in both moderate and severe asthmatic patients. While decorin staining was evident in the reticular basement membrane, it was minimal within the smooth muscle layer. Biopsy specimens from both moderate and severe asthmatics showed substantial amounts of smooth muscle. Only a small proportion of biopsies $(\sim 11 \%)$ contained negligible ASM.

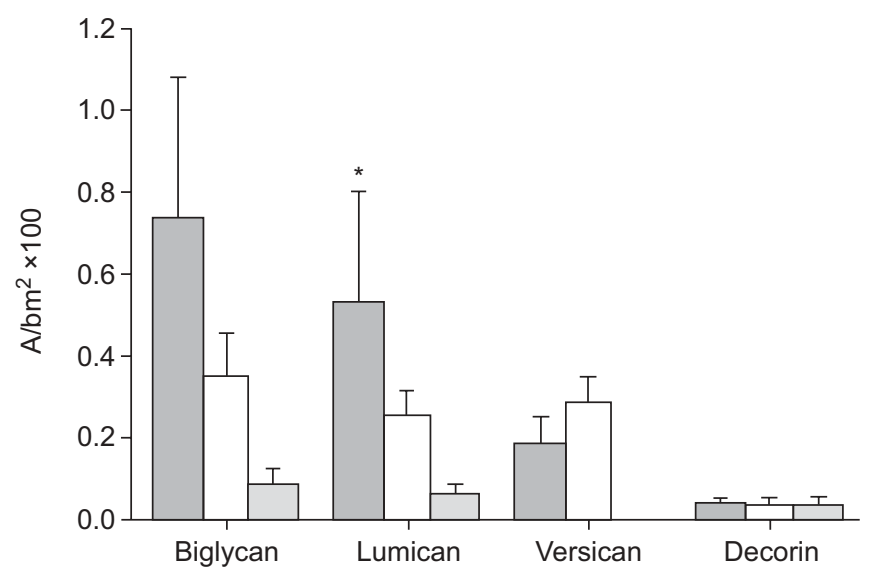

FIGURE 2. Area of positive staining in the subepithelial layer standardised for basement membrane length squared $\left(\mathrm{A} / \mathrm{bm}^{2}\right)$ for biglycan, lumican, versican and decorin in biopsy specimens from severe $(\square)$ and moderate $(\square)$ asthmatic patients, and control subjects $(\square)$. Data are presented as mean \pm SE. *: $p<0.05$ versus control subjects 


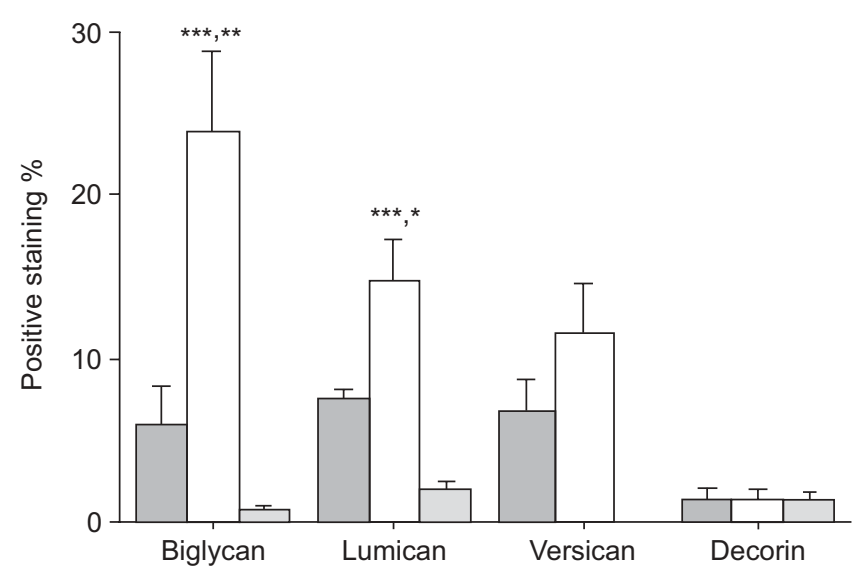

FIGURE 3. Percentage area of smooth muscle layer staining positive for biglycan, lumican, versican and decorin in biopsy specimens from severe ( $\square$ ) and moderate $(\square)$ asthmatic patients, and control subjects $(\square)$. Data are presented as mean+SE. *: $p<0.05$ versus severe asthmatic patients; ${ }^{* *}: p<0.01$ versus severe asthmatic patients; ***: $p<0.001$ versus control subjects.

\section{Morphometry}

Quantification of the area of positive staining in the subepithelial layer was normalised by basement membrane length squared. Measurements for biglycan, lumican, versican and decorin are shown in figure 2. Staining for lumican was significantly increased in severe asthmatics versus normal controls $(\mathrm{p}<0.05)$ and there was a strong trend for differences among the three groups for biglycan $(p=0.06$; Kruskal-Wallis test). There were no significant differences in immunostaining for decorin or versican among the moderate and severe asthmatic and control groups.

PG expression in the smooth muscle layer was measured by point counting and is shown in figure 3 . In the smooth muscle layer of moderate asthmatic patients, compared with severe asthmatics, there was significantly greater protein expression of biglycan and lumican ( $\mathrm{p}<0.01$ and $\mathrm{p}<0.05$, respectively) and normal controls (both $\mathrm{p}<0.001$; ANOVA). Minimal staining for decorin was found in the smooth muscle layer in airways from all three groups.

\section{DISCUSSION}

The results of the present study show that remodelling of the airway wall differs between moderate and severe asthmatics; while PG deposition in the subepithelial layer was similar in the two groups of asthmatic patients, moderate asthmatics had significantly greater PG deposition within the smooth muscle layer than severe asthmatics and normal controls. Such a distribution of matrix in the airway wall could potentially modulate airway narrowing in the moderate asthmatic group, by impeding ASM shortening. These data are consistent with the recently advanced hypothesis that remodelling of the airway wall in asthma could serve a protective role [15].

Enhanced deposition of matrix within the subepithelial layer of the asthmatic airway wall is well established [1,2]. Studies in asthmatics of all severities have shown prominent thickening of the reticular basement membrane and subepithelial fibrosis [1-3]. The present authors have shown in mild asthmatics that deposition of the PGs versican, biglycan and lumican was increased in the subepithelial layer of the airway wall compared with that of normal subjects [6]. DE KLUIJVER et al. [13] reported recently that, while biglycan was increased in the lamina propria of airways from mild asthmatic patients as compared to controls, decorin was decreased. ROBERTS [11] examined autopsy specimens of patients who died of asthma, and showed that the airway wall stained prominently for versican, biglycan and decorin; staining was particularly evident in the submucosa and around smooth muscle cells. DE MEDEIROs et al. [19] showed recently that in patients dying of fatal asthma, versican content in the internal area of both large and small airways was increased compared with controls. Some information is also available from in vitro studies. Fibroblasts obtained from endoscopic biopsies of airways of mild asthmatic patients demonstrated increased production of versican, perlecan and biglycan compared with cells isolated from normal controls [20]. The present authors have shown that message for decorin is upregulated in these same cells [21]. The current study corroborates these findings: expression of PGs was increased in the subepithelial layer in patients with asthma compared with controls. Staining for the PGs biglycan and lumican within the smooth muscle layer was significantly increased in patients with disease of moderate severity compared with controls.

The potential correlation between changes in airway structure and airway function in asthma has been addressed previously. The link between increased collagen deposition in the subepithelium and asthma severity has been examined, and the results have been conflicting. BENAYOUN et al. [7] described an increase in collagen type III immunostaining in the subepithelial layer of the airways only in asthmatics with severe disease; however, $\mathrm{CHU}$ et al. [3] were unable to document an association between submucosal collagen deposition and degree of disease. A previous study by the current authors, in which moderate and severe asthmatics were grouped together, showed relatively greater amounts of submucosal collagen deposition in these patients, compared with subjects with mild disease and normal controls [5]. This observation is further addressed by recent results in a group of patients overlapping those of the current study [18]. While the percentage of subepithelial fibrosis was no different between moderate and severe asthmatics, the distance between the ASM and the epithelium was significantly decreased in severe asthmatic patients. ASM mass was also greater in severe patients. However, changes in the extracellular matrix at the level of the smooth muscle and the relation to disease severity have not been examined previously.

The present authors have previously shown a significant correlation between PG deposition in the subepithelial layer of the airway wall and clinical disease [6]. A significant correlation was determined between subepithelial deposition of PG and airways responsiveness in mild atopic asthmatics. Similarly, WESTERGREN-THORSSON et al. [20] showed in fibroblasts isolated from endoscopic biopsies that cells from asthmatic patients with the greatest degree of hyperresponsiveness produced larger amounts of PGs.

The current finding that patients with more moderate asthma have greater PG deposition within the smooth muscle layer than severe asthmatics is perhaps surprising. There is little 
published information on PGs in ASM in control subjects. The results of the current study demonstrate minimal deposition of PGs within the ASM layer under nondisease conditions. The potential effects of increased PG levels within the ASM layer can best be understood by considering theoretical models [14, $22,23]$. Generally, the accepted idea has been that increased matrix deposition in the airway wall would lead to increased airway constriction; a thickened submucosal layer would result in a greater degree of luminal narrowing for a given degree of ASM constriction. More recently, this hypothesis has been countered by the argument that increased matrix deposition within the smooth muscle layer could have a modulating effect $[15,16,24]$. Excessive matrix could increase the impedence to ASM constriction and thereby decrease the actual length change in the smooth muscle for a given degree of contractile stimulation. Along these lines, BRAMLEY et al. [25] demonstrated that excised tracheal strips exposed to proteolytic digestion of the matrix showed enhanced shortening in response to contractile stimulation. A recent study by NIIMI et al. [8] showed that airway wall thickening, measured by computer tomography, correlated negatively with airway reactivity in patients with asthma. The current data, showing greater matrix deposition within the smooth muscle layer in asthmatics in whom clinical disease was less severe, are the first direct evidence in patients to support the hypothesis that matrix deposition within the ASM layer might modulate disease severity. Further evidence can be drawn from a recent autopsy study in fatal asthmatics by DE MEDEIROS et al. [19]. While these authors did not specifically quantify PGs within the ASM layer, airway photomicrographs from fatal asthmatic patients showed a conspicuous absence of PG immunostaining in the ASM layer.

Unfortunately, in the current study, it was not possible to measure airway responsiveness in the two groups of patients, because of the severity of their underlying disease and the degree of baseline airway narrowing. It would have been interesting to correlate responsiveness with the amount of PG deposition within the ASM layer.

One potential confounding factor relates to the amount of steroid therapy received by the two groups of patients. While steroids could potentially affect PG deposition [26], there is no a priori reason to expect that steroids would differentially affect deposition in the subepithelial versus smooth muscle layer.

PGs are likely candidates to affect the mechanical impedance of the ASM layer. PGs consist of a protein core to which GAG side-chains are attached. These hydrophilic side-chains affect tissue turgor by altering the water content of the tissues and thereby the mechanical behaviour of the system [9]. In addition, PGs modulate collagen fibrillogenesis, and may affect tissue mechanics via effects on collagen fibril formation [9]. The present authors have conducted experiments in both in vivo and in vitro models, which demonstrate that alterations in PGs affect tissue viscoelastic behaviour. Experiments in isolated parenchymal strips showed that specific degradation of PGs altered tissue viscoelastic behaviour [27]. Both tissue elastance and resistance were decreased after digestion of GAG side-chains. In in vivo experiments on mice deficient in decorin, the effects of lack of decorin on the mechanical characteristics of both the parenchymal tissues and airway mechanics were examined. Lung compliance was increased and airway resistance decreased [28]. Hence, changes in PGs in the asthmatic airway wall are likely to result in alterations in the mechanical behaviour of the airway. Whether that alteration is in a positive or negative direction may depend on the specific layer of the airway wall in which deposition is enhanced.

A further question is the effect of PGs on the functional or phenotypic characteristics of the adjacent structural cells. Again, some information is available from in vitro studies. HIRST et al. [29] have shown that various ECM proteins, such as collagen and fibronectin, have the capacity to affect ASM proliferation. JOHNSON et al. [30] reported that ECM proteins secreted by asthmatic ASM, which are likely to include PG, enhance ASM proliferation, in an autocrine fashion. The precise effects of PG on ASM proliferation and/or contractility remain to be determined.

In conclusion, the results of the present study show that moderate asthmatic patients have relatively greater deposition of proteoglycan in the smooth muscle layer of the airway wall than do patients with more severe disease. This enhanced deposition of matrix could potentially serve a modulating role, protecting against excessive smooth muscle constriction. It could also impact on the phenotypic behaviour of the smooth muscle itself. These are some of the first data collected from patients which support the hypothesis that airway remodelling in asthma may be a "friend, not a foe" [15].

\section{REFERENCES}

1 Jeffery PK. Remodeling in asthma and chronic obstructive lung disease. Am J Respir Crit Care Med 2001; 164: S28-S38.

2 Roche WR, Beasley R, Williams JH, Holgate ST. Subepithelial fibrosis in the bronchi of asthmatics. Lancet 1989; 1: 520-524.

3 Chu HW, Halliday JL, Martin RJ, Leung DY, Szefler SJ, Wenzel SE. Collagen deposition in large airways may not differentiate severe asthma from milder forms of the disease. Am J Respir Crit Care Med 1998; 158: 1936-1944.

4 Laitinen A, Altraja A, Kampe M, Linden M, Virtanen I, Laitinen LA. Tenascin is increased in airway basement membrane of asthmatics and decreased by an inhaled steroid. Am J Respir Crit Care Med 1997; 156: 951-958.

5 Chakir J, Shannon J, Molet S, et al. Airway remodelingassociated mediators in moderate to severe asthma: effect of steroids on TGF- $\beta$, IL-11, IL-17, and type I and type III collagen expression. J Allergy Clin Immunol 2003; 111: 1293-1298.

6 Huang J, Olivenstein R, Taha R, Hamid Q, Ludwig MS. Enhanced proteoglycan deposition in the airway wall of atopic asthmatics. Am J Respir Crit Care Med 1999; 160: 725-729.

7 Benayoun L, Druilhe A, Dombret MC, Aubier M, Pretolani M. Airway structural alterations selectively associated with severe asthma. Am J Respir Crit Care Med 2003; 167: 1360-1368.

8 Niimi A, Matsumoto H, Takemura M, Ueda T, Chin K, Mishima M. Relationship of airway wall thickness to airway sensitivity and airway reactivity in asthma. Am J Respir Crit Care Med 2003; 168: 983-988.

9 Iozzo RV. Matrix proteoglycans: from molecular design to cellular function. Annu Rev Biochem 1998; 67: 609-652. 
10 Ruoslahti E, Yamaguchi Y. Proteoglycans as modulators of growth factor activities. Cell 1991; 64: 867-869.

11 Roberts CR. Is asthma a fibrotic disease? Chest 1995; 107: Suppl. 3, 111S-117S.

12 Redington AE, Roche WR, Holgate ST, Howarth PH. Colocalization of immunoreactive transforming growth factor-beta 1 and decorin in bronchial biopsies from asthmatic and normal subjects. J Pathol 1998; 186: 410-415.

13 de Kluijver J, Schrumpf JA, Evertse CE, et al. Bronchial matrix and inflammation respond to inhaled steroids despite ongoing allergen exposure in asthma. Clin Exp Allergy 2005; 35: 1361-1369.

14 Moreno RH, Hogg JC, Pare PD. Mechanics of airway narrowing. Am Rev Respir Dis 1986; 133: 1171-1180.

15 McParland BE, Macklem PT, Pare PD. Airway wall remodeling: friend or foe? J Appl Physiol 2003; 95: 426-434.

16 Pare PD. Airway hyperresponsiveness in asthma: geometry is not everything!. Am J Respir Crit Care Med 2003; 168: 913-914.

17 American Thoracic Society, Proceedings of the ATS workshop on refractory asthma: current understanding, recommendations, and unanswered questions. Am J Respir Crit Care Med 2000; 162: 2341-2351.

18 Pepe C, Foley S, Shannon J, et al. Differences in airway remodeling between subjects with severe and moderate asthma. J Allergy Clin Immunol 2005; 116: 544-549.

19 de Medeiros MM, da Silva LF, dos Santos MA, et al. Airway proteoglycans are differentially altered in fatal asthma. J Pathol 2005; 207: 102-110.

20 Westergren-Thorsson G, Chakir J, Lafrenière-Allard MJ, Boulet LP, Tremblay GM. Correlation between airway responsiveness and proteoglycan production by bronchial fibroblasts from normal and asthmatic subjects. Int $J$ Biochem Cell Biol 2002; 34: 1256-1267.
21 Ludwig MS, Ftouhi-Paquin N, Huang W, Page N, Chakir J Hamid Q. Mechanical strain enhances proteoglycan message in fibroblasts from asthmatic subjects. Clin Exp Allergy 2004; 34: 926-930.

22 Lambert RK, Wiggs BR, Kuwano K, Hogg JC, Pare PD. Functional significance of increased airway smooth muscle in asthma and COPD. J Appl Physiol 1993; 74: 2771-2781.

23 Wiggs BR, Hrousis CA, Drazen JM, Kamm RD. On the mechanism of mucosal folding in normal and asthmatic airways. J Appl Physiol 1997; 83: 1814-1821.

24 Macklem PT. A theoretical analysis of the effect of airway smooth muscle load on airway narrowing. Am J Respir Crit Care Med 1996; 153: 83-89.

25 Bramley AM, Roberts CR, Schellenberg RR. Collagenase increases shortening of human bronchial smooth muscle in vitro. Am J Respir Crit Care Med 1995; 152: 1513-1517.

26 Todorova L, Gurcan E, Miller-Larsson A, WestergrenThorsson G. Lung fibroblast proteoglycan production induced by serum is inhibited by budesonide and formoterol. Am J Respir Cell Mol Biol 2006; 34: 92-100.

27 Al Jamal R, Roughley PJ, Ludwig MS. Effect of glycosaminoglycan degradation on lung tissue viscoelasticity. Am J Physiol Lung Cell Mol Physiol 2001; 280: L306-L315.

28 Fust A, LeBellego F, Iozzo RV, Roughley PJ, Ludwig MS. Alterations in lung mechanics in decorin-deficient mice. Am J Physiol Lung Cell Mol Physiol 2005; 288: L159-L166.

29 Hirst SJ, Twort CH, Lee TH. Differential effects of extracellular matrix proteins on human airway smooth muscle cell proliferation and phenotype. Am J Respir Cell Mol Biol 2000; 23: 335-344.

30 Johnson PR, Burgess JK, Underwood PA, et al. Extracellular matrix proteins modulate asthmatic airway smooth muscle cell proliferation via an autocrine mechanism. J Allergy Clin Immunol 2004; 113: 690-696. 